\title{
ECG imaging of ventricular tachycardia: evaluation against simultaneous non-contact mapping and CMR-derived grey zone
}

\author{
Walther H. W. Schulze ${ }^{1} \cdot$ Zhong Chen $^{3} \cdot$ Jatin $\operatorname{Relan}^{4} \cdot$ Danila Potyagaylo $^{1} \cdot$ \\ Martin W. Krueger ${ }^{1,2} \cdot$ Rashed Karim $^{3} \cdot$ Manav Sohal $^{3}$ - Anoop Shetty ${ }^{3}$. \\ YingLiang $\mathrm{Ma}^{3} \cdot$ Nicholas Ayache $^{4} \cdot$ Maxime Sermesant $^{4} \cdot$ Herve Delingette $^{4}$. \\ Julian Bostock ${ }^{3} \cdot$ Reza Razavi $^{3}$ Kawal S. Rhode ${ }^{3}$ Christopher A. Rinaldi ${ }^{3}$. \\ Olaf Dössel ${ }^{1}$
}

Received: 31 December 2015/Accepted: 2 September 2016/Published online: 20 September 2016

(C) International Federation for Medical and Biological Engineering 2016

\begin{abstract}
ECG imaging is an emerging technology for the reconstruction of cardiac electric activity from non-invasively measured body surface potential maps. In this case report, we present the first evaluation of transmurally imaged activation times against endocardially reconstructed isochrones for a case of sustained monomorphic ventricular tachycardia (VT). Computer models of the thorax and whole heart were produced from MR images. A recently published approach was applied to facilitate electrode localization in the catheter laboratory, which allows for the acquisition of body surface potential maps while performing non-contact mapping for the reconstruction of local activation times. ECG imaging was then realized using Tikhonov regularization with spatio-temporal smoothing as proposed by Huiskamp and Greensite and further with the spline-based approach by Erem et al. Activation times were computed from transmurally reconstructed transmembrane voltages. The results showed good qualitative agreement between the non-invasively and
\end{abstract}

The research leading to these results was co-funded by the European Commission within the Seventh Framework Programme (FP7/20072013) under Grant Agreement No. 224495 (euHeart project) and by the German Research Foundation under grants DO637/10-1 and DO637/13-1.

Walther H. W. Schulze

publications@ibt.kit.edu

1 Institute of Biomedical Engineering, Karlsruhe Institute of Technology (KIT), Kaiserstr. 12, 76131 Karlsruhe, Germany

2 Present Address: ABB AG, Corporate Research, Ladenburg, Germany

3 Division of Imaging Sciences, St. Thomas' Hospital, King's College London, London, UK

4 INRIA Sophia Antipolis, Sophia Antipolis, France invasively reconstructed activation times. Also, low amplitudes in the imaged transmembrane voltages were found to correlate with volumes of scar and grey zone in delayed gadolinium enhancement cardiac MR. The study underlines the ability of ECG imaging to produce activation times of ventricular electric activity-and to represent effects of scar tissue in the imaged transmembrane voltages.

Keywords Inverse problem of ECG - Clinical validation . Ventricular tachycardia $\cdot$ Non-contact mapping $\cdot$ ECG imaging

\section{Introduction}

ECG imaging is an evolving technology for the non-invasive reconstruction of cardiac electric activity from body surface potential maps (BSPMs), which is well introduced and reviewed in [36, Chapt. 9] as the inverse electrocardiographic problem. We present an application of this technique for the imaging of transmembrane voltages (TMVs) and related activation times in case of monomorphic ventricular tachycardia (VT). With conventional noninvasive methods it is a clinical challenge and time-consuming procedure to localize exit points and pathways of VT [70], which represent necessary information for therapy planning and guidance in radio-frequency ablation.

While several validations of ECG imaging have been performed in animal studies (see [4, 5, 21, 22] for VT), validation in humans is challenging [41], especially for the ventricles. Some animal studies work with the heart placed in a torso tank $[4,5]$, which is a strongly simplified model of the inhomogeneous, anisotropic and largely unknown [30] conductivities of the human torso. When 
measuring with complete thorax models in closed-chest conditions [21, 22], validation results can be considered very realistic in terms of performance (exit site localization errors were $5 \mathrm{~mm}$ in [21], $7 \mathrm{~mm}$ in [22]). The interpretation of these two works for application in humans, however, is limited due to the species-specific anatomies and the experiment set-ups, which allowed for controlled breathing and the by-passing of clinical restrictions (radiation use in model generation, ability to apply electrodes in the CT images and keep them in place during the ECG imaging study). The great scientific value of animal studies on the other hand is that validation data can be obtained from electrode socks that are placed directly on the epicardium, which requires open chest surgery [4, 5, 21, 22].

Only a few studies exist on the localization of excitation origins or low-voltage areas in humans, where validation data have to be obtained from invasive electroanatomical mapping. In particular, epicardial electrograms have been reconstructed to identify the origins of pacings in $[27,50,54]$, endo- and epicardial electrograms were imaged simultaneously to identify origins in a comprehensive pacing study in [14] or to identify WPW pathways in [3], ablation sites of premature ventricular contractions were localized on the endocardium in [33], and potentials of ischaemic areas were identified on the epicardium in [37]. In the latter publication, occlusion sites of percutaneous transluminal coronary angioplasty served as validation reference. A further validation study has been performed that evaluates ventricular epicardial electrograms of right ventricular (RV) pacing sites and normal sinus rhythm from an open chest surgery against ECG imaging from non-simultaneous BSPM measurements [18].

In this work, which forms part of a recently published thesis [57], we present the imaging of activation isochrones for a transient episode of monomorphic VT, which only sustained for an interval of several beats. The non-repeating nature of the event led to two requirements: activation times as reference reconstruction had to be produced from single beats and were provided by non-contact electroanatomical mapping. Second, the BSPM recordings had to take place during the electrophysiological study, which required the use of a novel electrode localization procedure [69].

To our knowledge, we present the first study for the imaging of VT, where transmural distributions of TMVs are reconstructed and evaluated in terms of their activation times in humans. Only two previous publications exist for ECG imaging of VT in humans [54, 63], as well as a case study in magnetocardiography [40]. In [63], ECG imaging results were produced for nine patients during sustained monomorphic VT, five of them with reentrant VT mechanisms as in the present work. However, results were only produced for the epicardium, while they were compared with electroanatomical maps of the endocardium (see limitations in [63]). Also, no quantitative analysis on the localization accuracy was produced, despite the good correspondence between invasive endocardial mapping and non-invasively reconstructed activation patterns. In the second work [54], the site of successful ablation or clinically identified exit point was used as validation reference. Results were presented for 2 cases of VT with epicardial exit sites. Localization accuracy of the exit sites was evaluated in terms of qualitative consistency. In this work, we produce quantitative features for the localization accuracy of the exit site and also for the agreement between endocardially reconstructed isochrones and noninvasively imaged activation patterns. We further produce sensitivities and specificities for an amplitude-based feature of the imaged transmembrane voltages that may be related to scars in the myocardial substrate.

\section{Methods}

Sustained monomorphic VT was induced in a patient in line with the 12 stage VT stimulation protocol by Wellens et al. [66]. This patient (KIT Patient 02) has previously been reported on in [7, Fig. 1a-g]. The study was performed for risk stratification in a clinical decision on whether to implant an implantable cardioverter defibrillator (ICD). All clinical data were collected at King's College London (KCL) and Guy's Hospital, London. The study was approved by the local ethics committees, and written informed consent was obtained from the patients. The overall study that led to this work included four other patients, three of which did not provide data of simultaneous non-contact mapping of VT and one of which was not evaluated.

A personalized computer model of the patient was produced. Then, ECG imaging was used to calculate transmembrane voltages from BSPMs, and corresponding activation times were derived. The non-invasively computed activation times were then compared with endocardially reconstructed activation times using a non-contact mapping system.

\subsection{Personalized patient model}

Magnetic resonance imaging was performed at Guy's Hospital, London, using a Philips Achieva 1.5T MRI scanner (Philips, Best, The Netherlands). Thorax scans were acquired with a SENSE breath-hold ultra-fast gradient echo sequence during expiration. MRI acquisitions of the whole heart were taken, including the atria. To this end, an ECG-gated SSFP sequence was used that was triggered by a navigator to add respiratory gating. The thorax MRI 
was segmented into distinct tissue classes for skeletal muscle, lungs, liver, kidneys, stomach, spleen and aorta. In the heart model, the left and right ventricle and the left and right atrium were considered, including specific classes for the pulmonary trunk and aorta, and the corresponding lumina were annotated as venous and arterial blood pools. Segmentation of the thorax was performed manually by Karlsruhe Institute of Technology (KIT) with the PHILIPS CHD segmentation package by Philips Research Hamburg, Germany. The software is a tool for quick, three-dimensional and adaptive generation of segmentations and uses a level set method [31]. The segmentation of the whole heart scan was produced automatically by KCL with the PHILIPS SSFP cardiac segmentation plug-in [13]. Ventricular scar tissue was segmented by KCL from cardiac magnetic resonance (CMR) images that were taken after contrast injection (delayed-enhancement). Scar core zone was segmented using the full-width-half-maximum method and scar grey zone (an admixture of scar tissue and healthy myocardium) was segmented with a cut-off signal intensity below that of the core and above 2 standard deviations of the remote healthy myocardium [7, 53]. From the segmented images of the heart and thorax, personalized tetrahedral meshes were generated using the computational geometry algorithms library (CGAL) [12]. Conductivities were assigned to the tissue classes according to [16].

\subsection{ECG imaging}

BSPMs were collected with the ActiveTwo recording system by BioSemi B.V., Amsterdam, the Netherlands, using 51 active electrodes which were placed on the anterior and posterior part of the patient thorax. The recordings were pre-processed using a wavelet-based approach to remove baseline-wander and high-frequency noise as in [32], and a notch filter was used to remove 50-Hz noise.

To perform ECG imaging, TMVs were used as a source model [24, 34, 42, 46, 56, 62] and the related BSPMs for TMV sources in a volumetric finite element grid of the ventricles were computed under quasistatic assumptions using the bidomain model [17]. Calculations on the personalized patient geometry as in [45] yield a linear formulation of the forward problem that relates the TMVs in the heart $x \in \mathbb{R}^{n}(n=2346)$ to the potentials at $m$ BPSM electrodes $b \in \mathbb{R}^{m}(m=51)$, where $A \in \mathbb{R}^{m \times n}$ is the lead field matrix:

$A x=b$

The problem was solved for a time sequence $X=\left[x_{1}, x_{2}, \ldots, x_{\tau}\right], B=\left[b_{1}, b_{2}, \ldots, b_{\tau}\right]$ using two methods: the spatio-temporal approach by Huiskamp and
Greensite [20] and the spline-based approach by Erem et al. [14]. First, for the approach by Huiskamp and Greensite, the spatio-temporal problem was formulated as

$$
\begin{aligned}
& A X=B=\mathrm{USV}^{T}, \\
& \Leftrightarrow A(X V)=B V
\end{aligned}
$$

where $\mathrm{USV}^{T}$ is the singular value decomposition of $B$. For the first $p$ components in $V$

$$
A(X \tilde{V})=B \tilde{V}, \quad \tilde{V} \in \mathbb{R}^{\tau \times p}
$$

the inverse problem of ECG can be solved efficiently for $\left(X \tilde{V}_{i}\right), i \in 1, \ldots, p$, using the Tikhonov method ( $L$ Laplace operator),

$$
\left(X \tilde{V}_{i}\right)_{\lambda}=\underset{\left(X \tilde{V}_{i}\right)}{\operatorname{argmin}}\left\|A\left(X \tilde{V}_{i}\right)-B \tilde{V}_{i}\right\|_{F}+\lambda\left\|L\left(X \tilde{V}_{i}\right)\right\|_{F},
$$

and the resulting solution can be transformed back into the temporal domain

$$
X_{\lambda}=(X \tilde{V})_{\lambda} \tilde{V}^{T}
$$

While an identification of the regularization parameter $\lambda$ with the L-curve method [23] resulted in an L-curve corner at $10^{-4}$ for the Greensite method, significantly better results were obtained with $\lambda=10^{-3}$, which was chosen for the final evaluation. Truncation parameter $p$ was set to 10 in line with the finding in [25], which suggests that the number of independent singular vectors in a BSPM is of the order of 10. Robustness against parameter variations in $\lambda$ and $p$ was then thoroughly evaluated for the present case in Fig. 3 to demonstrate the effect of this choice.

Spatio-temporal regularization with the method by Huiskamp and Greensite is well suited for stable reconstruction across the time points of a VT cycle, compared to methods that identify focal activity or that operate on single time points [46, 49, 50]. Further, it is better suited for cases with large scars compared to spatio-temporal methods that make rigorous assumptions on action potential shapes and tissue excitability, such as rule-based approaches [47] or the critical times method [26]. On the other hand, methods which require that TMV courses are non-decreasing in time [11, 39] impose spatio-temporal constraints that leave room for behaviour associated with scar tissue, but they must make the assumption that electric sources from repolarization are negligible during VT cycles. Despite its advantages over these methods, the approach by Huiskamp and Greensite may be criticized for its underlying assumption that the temporal and the spatial behaviour of the reconstructed signals are decoupled [14], which is clearly not the case for cardiac excitation wavefronts. 
Therefore, Erem et al. [14] proposed a spline-based approach that only makes the assumption that the temporal and the spatial behaviour are decoupled locally in time. The method approximates the BSPM time sequence $B$ with a spline curve that is smooth in the high-dimensional space of its electrode potentials and parameterized by a predetermined number of knot points at irregular temporal intervals. Next, it solves the inverse problem at the knot points for TMVs with a Tikhonov-based solver that is parameterized using the L-curve method [23]. Finally, it reconstructs the TMV time sequence $X$ based on the temporal dynamics of the BSPM approximation. In this work, we use an implementation of the method as provided with the toolkit for inverse problems in electrocardiography of the SCIRun problem-solving environment $[6,10,58]$. In the work of Erem et al. [14] the number of knots was consistently set to 12 , and results were found to be robust to the choice of this parameter. The evaluated VT beat has a number of 272 time samples, which is on the order of 300 as in the PVC beats of [14]. We therefore deduced that we could use the same number of knots, and we further evaluated results for 14 and 16 knots (see Fig. 3) to account for the potentially more complex temporal dynamics of the VT beat.

For both methods, results were scaled to range from -85 to $15 \mathrm{mV}$ in each time step to be better suited for interpretation and post-processing. Without the measure, TMV time courses in the inverse solutions differed only slightly or even marginally (Greensite method) between different nodes. An example of a reconstructed time course after scaling is shown for a single heart node of the RVOT in Fig. 1 for both of the methods.

For validation, reconstructed TMVs can only be indirectly evaluated in terms of assigned activation times (ATs), or alternatively in terms of their computed

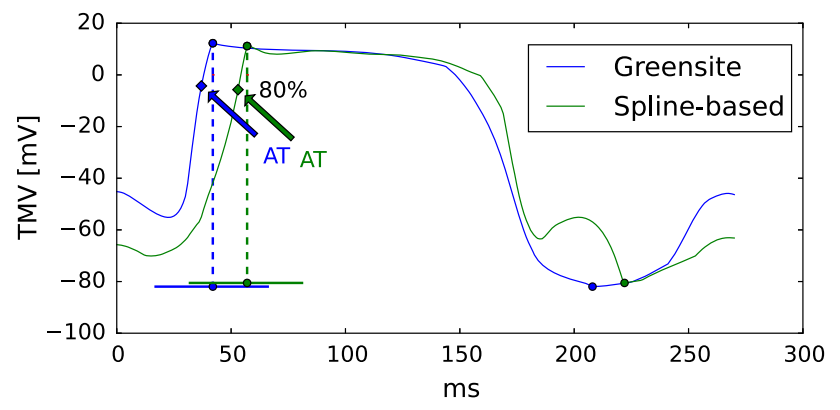

Fig. 1 The first time when a reconstructed TMV time course crosses $80 \%$ of its amplitude (arrows) was defined as activation time (AT). The amplitude itself (dashed lines) was used as a feature to detect scar or grey zone tissue. In this example of a single heart node in the RVOT, the TMV time courses were reconstructed with the Greensite method $\left(\lambda=10^{-3}, p=10\right)$ and the spline-based method (14 knots). The TMVs were scaled in each time step to range from -85 to $15 \mathrm{mV}$, i.e. the range was enforced across the ventricles electrograms (optical mapping with voltage-sensitive fluorescence dye is not available in humans in vivo, see $[44,65]$ for a study in ex-vivo porcine hearts). After solving the inverse problem for the VT beat in Fig. 2a, activation times were obtained from the reconstructed TMVs of the evaluated beat.

To assign an activation time to each heart node in the non-invasive reconstruction, we identified the first time when its reconstructed TMV time course crosses $80 \%$ of its amplitude, see arrows in the illustration of Fig. 1. The amplitudes are shown here as dashed lines, illustrating that for each node, the amplitude was defined as the difference between the maximum and minimum TMV in the time course. All activation times were produced with respect to the time of the presumed VT wave exit at $0 \mathrm{~ms}$ (see solid line in Fig. 2a), which was assumed to correspond to the reference in the activation isochrones of the non-contact mapping reconstruction.

Further, as a feature for detection of scar or grey zone tissue, nodes with low amplitudes were identified. For classification of low-amplitude areas as scar tissue an upper amplitude threshold was set. Results were then validated against the CMR-derived scar core and grey zone. To avoid an empirical parameterization and acknowledging that the reference scar and grey zone segmentations were originally threshold dependent as well, we decided to couple the upper amplitude threshold directly to the given segmentations: the amplitude threshold $(64 \mathrm{mV}$ for the Greensite method, $60 \mathrm{mV}$ for the spline-based method) was chosen such that the low-amplitude areas had the same coverage of the left ventricle as the scar and grey zone areas from CMR. The coverage was calculated in terms of nodes in the heart geometry, i.e. nodes that had been classified as having low-amplitude action potentials or that had been assigned scar or grey zone tissue, respectively.

\subsection{Non-contact mapping}

For validation of ECG imaging, catheter measurements in direct contact with the myocardial wall have been the standard since electroanatomical mapping became available [41]. This works well with single-point catheter measurements from multiple beats, given that the cardiac event can be measured repeatedly at different positions. In that case the single-point measurements can be merged into well-covered local activation time maps [61].

In the present study, however, the measured monomorphic VT sustained only for a short period of time before it terminated. To record activation isochrones of the arrhythmic activity at once, non-contact mapping with a multi-electrode array (MEA) was used [15, 43]. From the MEA measurements activation maps were computed with the EnSite ${ }^{\circledR}$ system $[19,55,67]$ of St. Jude Medical, Inc., 


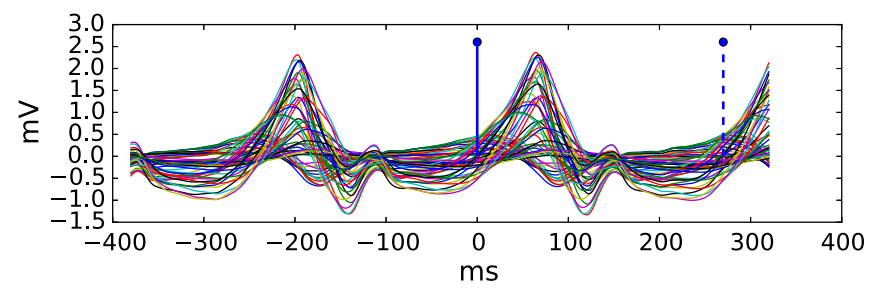

(a)
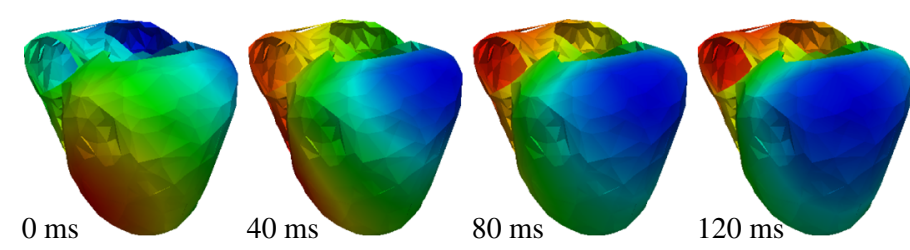

(c)
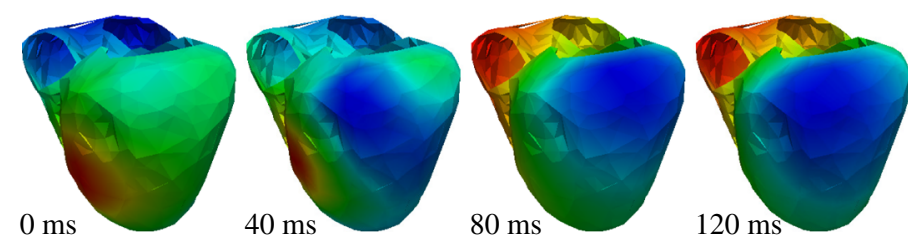

(d)

Fig. 2 Sustained monomorphic VT: evaluation of ECG imaging against simultaneous non-contact mapping (LV/RV: left/right ventricle). a Sustained monomorphic VT: ECGs as measured with the 51-channel BSPM mapping system. The evaluated interval of the beat starts at $0 \mathrm{~ms}$ (solid line) and ends at $271 \mathrm{~ms}$ (dashed line), b EnSite ${ }^{\circledR}$

for a simplified shell of the endocardium. The MEA was positioned in the left ventricular (LV) cavity, and the resulting endocardial shell was registered with the CMRbased model of the heart using anatomical landmarks [69]. To this end, the roving catheter was moved to five locations within the LV and its coordinates were recorded in the EnSite $^{\circledR}$ and X-ray coordinate systems. This allowed for a rigid transformation to be computed between theses coordinate systems. The MR data and therefore the CMR-based model were registered to the X-ray coordinate system using the XMR method described in [52].

To obtain the endocardial shell the EnSite ${ }^{\circledR}$ system estimates the LV geometry with a contoured-geometry approach from locator signals that are emitted by a catheter, which is moved to trace the endocardial surface of the LV [28]. Then, the system localizes the MEA catheter, which is also emitting locator signals. The accuracy of this localization was assessed in [28] and found to be highly accurate. A boundary element method model of the LV is then used to reconstruct virtual electrograms on the endocardial shell by solving the ill-posed inverse problem of ECG for the endocardium. The EnSite ${ }^{\circledR}$ system then
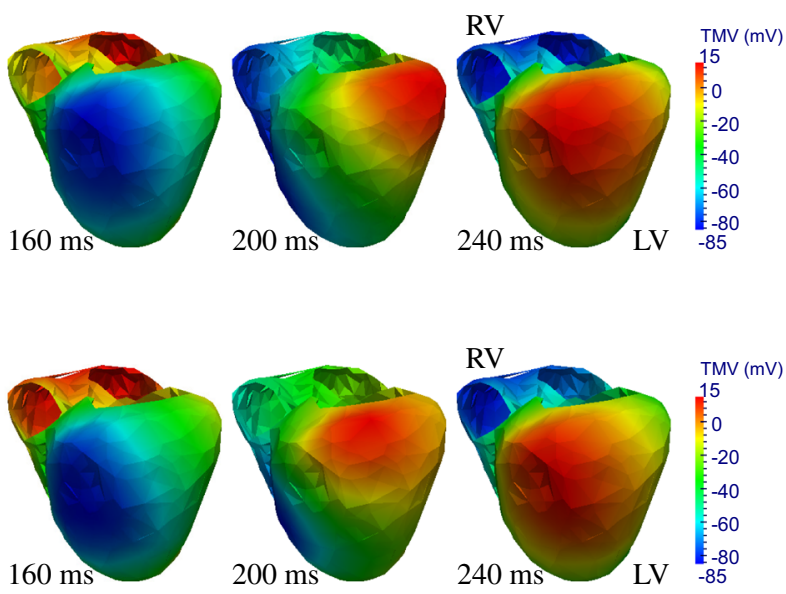

Greensite method Spline-based

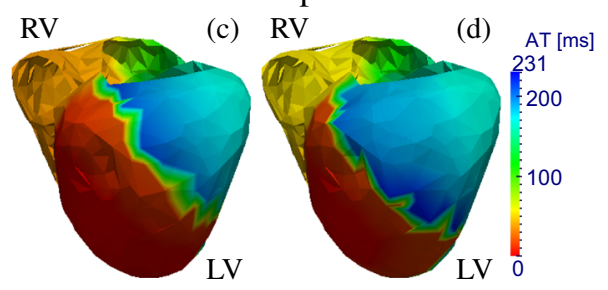

(b)

activation isochrones of the LV endocardial surface (left). Activation times from ECG imaging of the LV and RV volume: Greensite method (middle), spline-based method (right), c Greensite method: TMVs of the evaluated VT beat, $\mathbf{d}$ spline-based method: TMVs of the evaluated VT beat

computes activation times from these virtual electrograms for the endocardial shell, which were used as an evaluation reference for the non-invasive mapping in this study.

To this end, electroanatomical mapping was performed simultaneously with the recording of the BSPM. This required the use of BSPM electrode localization in the cath lab. BSPM electrodes were therefore localized using a biplane X-ray-based localization system with $0.33 \pm$ $0.20 \mathrm{~mm}$ accuracy [69] and rigidly co-registered with the thorax MRI using multi-modality markers.

\section{Results}

ECG imaging was performed to reconstruct TMVs in the myocardial volume of the ventricles by processing the BSPM signals of the annotated beat of the sustained monomorphic VT in Fig. 2a. Results for the beat, which starts at the blue solid line and ends at the dashed line, are shown in Fig. $2 \mathrm{c}$ for the Greensite method with $\lambda=$ $10^{-3}, p=10$ and for the spline-based method (14 knots) in Fig. 2 d. The sequences clearly show a wavefront of TMVs 
in the depolarized range (red) that rotates from the anterolateral side of the left ventricle (LV) over its anterior side towards the right ventricle (RV) and then over the posterior side back towards the anterolateral wall of the LV.

This is reflected in the activation times obtained from the inverse solutions, which are displayed in Fig. $2 b$ for the $\mathrm{RV}$ and LV and range from 0 to $231 \mathrm{~ms}$ for the Greensite method and from 0 to $227 \mathrm{~ms}$ for the spline-based method (parameters were again $\lambda=10^{-3}, p=10$ and 14 knots, respectively). The shell of the LV endocardial surface to the left shows the isochrones obtained from invasive mapping with the MEA system, which range up to only $123 \mathrm{~ms}$. The shell is registered with the CMR-based model of the ECG imaging solution to reveal the qualitative agreement between the activation patterns.

The qualitative agreement was quantitatively analysed for the endocardial surface: each node in the EnSite ${ }^{\circledR}$ shell was assigned its closest node in the FEM mesh for the noninvasive calculations. Activation times of these nodes were then compared: Table 1 shows the correlation coefficient and the root mean squared error (RMSE) between the activation times at the closest nodes as well as the distance between the excitation origins (mean position of those $25 \%$ of the nodes in a map that have the earliest activation times). Figure 3 demonstrates that for the Greensite method, the correlation coefficient of $66 \%$ as well as the distance between the excitation origins of $5.4 \mathrm{~mm}$ were strongly affected by the choice of the spatial regularization parameter $\lambda$, yet only slightly affected by the choice of the truncation parameter $p$. For the spline-based method, where the spatial regularization parameter was chosen automatically, Fig. 3 reveals that correlation coefficients were in the range of the best correlation coefficients of the Greensite method. The best correlation coefficient of $63 \%$ was obtained for 14 knots with a distance between the excitation origins of $8.5 \mathrm{~mm}$. Just as for the truncation parameter of the Greensite method, results with the spline-based method were little sensitive to variations in the number of
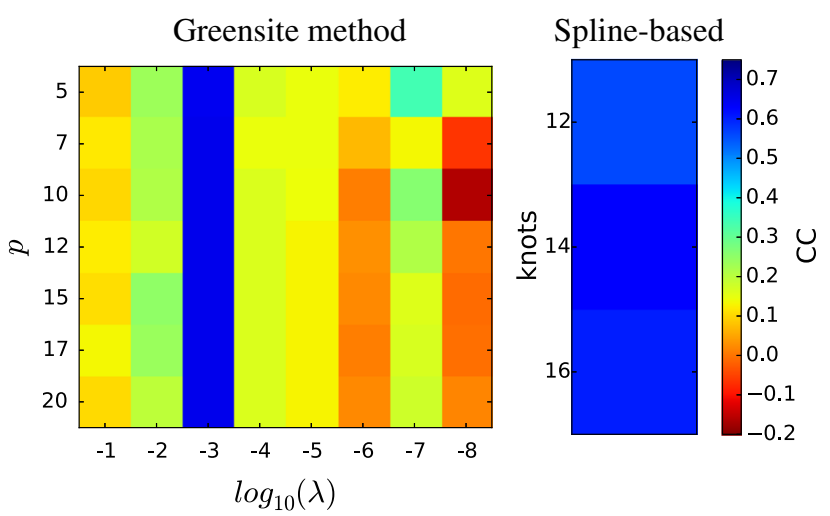

(a)

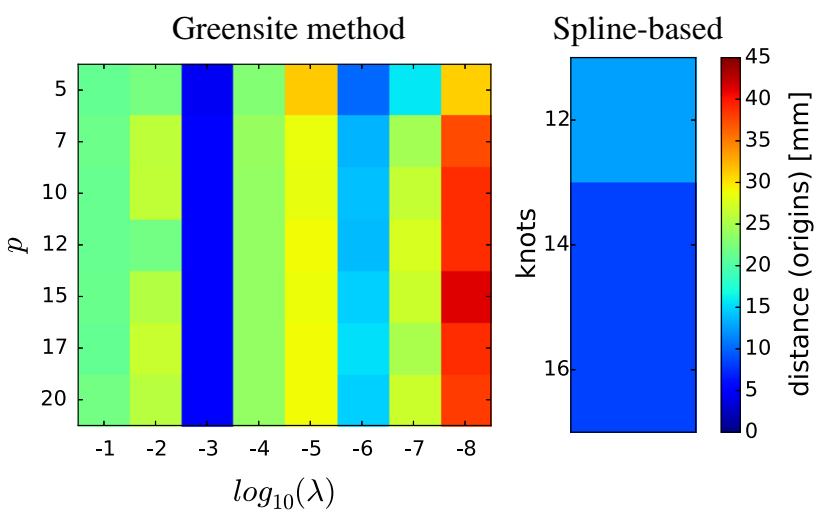

(b)

Fig. 3 Robustness against solver parameters $\lambda, p$, knots: correlation of the activation times of the EnSite ${ }^{\circledR}$ system (endocardial shell) with activation times at the closest nodes in the reconstruction (top), distance between excitation origins (mean positions of the earliest $25 \%$, bottom). a Correlation coefficient, b distance between excitation origins

knots, with a correlation of $56 \%$ for 12 knots and a correlation of $60.4 \%$ for 16 knots. As the spatial regularization parameter for the spline-based method was chosen with the L-curve method, the study does not include a demonstration of its variation. Figure 3 is therefore not intended to be an assessment of the general robustness of
Table 1 Correspondence between activation times of the invasive mapping and activation times from the ECG imaging solutions

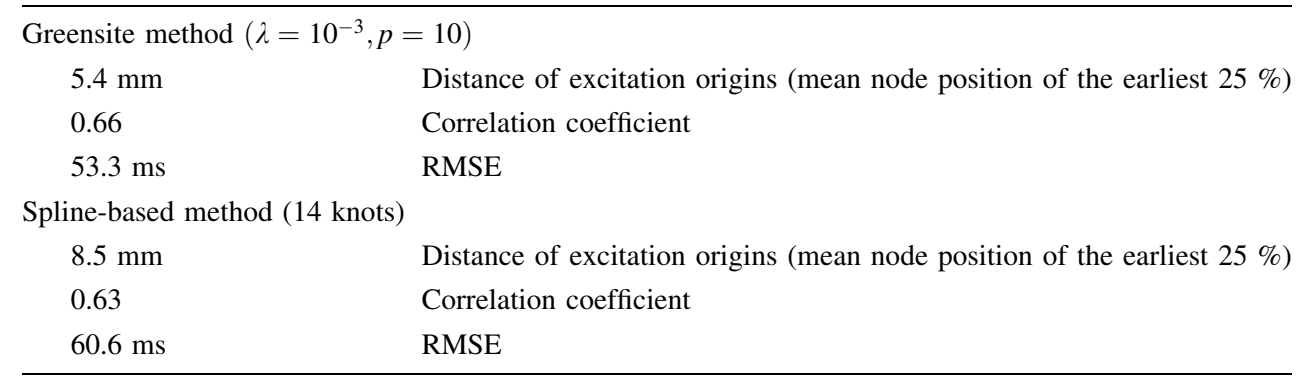

The distance of excitation origins is shown as well as the correlation coefficient and the root mean squared error (RMSE). For the evaluation, each node in the EnSite ${ }^{\circledR}$ shell was assigned its closest node in the FEM mesh of the non-invasive reconstructions 
Fig. 4 Activation times (ATs) from the EnSite ${ }^{\circledR}$ mapping system and from the Greensite method are superimposed over CMR-derived scar (black) and grey zone (grey) volumes in (a). For the LV and for the same view, the correspondence between low-amplitude areas in the ECG imaging reconstructions and scar is shown in (b). To this end, the LV is split into its septal and its lateral half. a View of the LV with CMR-derived scar core and grey zone: activation isochrones from EnSite ${ }^{\circledR}$ (left) and from ECG imaging with the Greensite method (right), b septal half and lateral half of the view in (a): LV scar and grey zone (top) as detected with the Greensite method (middle) and the spline-based method (bottom)

both methods in terms of their regularization parameters. Instead, it is intended to show the effect of all regularization parameters which have been picked manually in this work ( $\lambda, p$, number of knots).

For the same parameterizations that were chosen in Fig. $2\left(\lambda=10^{-3}, p=10\right.$ and 14 knots, respectively), a statistical evaluation was conducted for the LV to understand the correlation between CMR-derived scar or grey zone and low amplitudes in the non-invasively imaged TMV time courses. In Fig. 4a, CMR derived scar is superimposed over the EnSite ${ }^{\circledR}$ activation isochrones (left) and the activation times obtained with the Greensite method (right), where the LV is shown from the same lateral view as in Fig. 2b. In Fig. 4b, the view is split in its septal and lateral halves. Myocardial volumes of the LV where the reconstructed TMV time course did not exceed the predetermined amplitude threshold $(64 \mathrm{mV}$ for the Greensite method, $60 \mathrm{mV}$ for the spline-based method) were compared to scar volumes from CMR (top). Where these volumes coincided, low amplitudes did correctly (T: true) predict scar or grey zone in the tissue (P: positivescar or grey zone, N: negative-healthy substrate according to scar segmentation). It is revealed that low-amplitude areas in the Greensite reconstruction (middle) corresponded well with low-amplitude areas in the spline-based reconstruction (bottom). Also, the low-amplitude areas of both approaches covered most of the areas of the CMRderived scar and grey zone tissue (TP: true positive), except for the basal part of the posterior scar. This is reflected in a sensitivity $(T P / P)$ of $56 \%$ for the LV volume in case of the Greensite method and a sensitivity of $45 \%$ in case of the spline-based method (see Table 2). While the apical part of the LV was largely classified false positive with both methods, it is apparent that in almost the entire lateral half where no low amplitudes were detected there was also little scar tissue or grey zone present (TN: true negative). This is represented in a specificity ( $T N /$ $N$ ) of $70 \%$ for the Greensite method and $66 \%$ for the spline-based method.

Specificities increased to $87 \%$ for the Greensite method and to $81 \%$ for the spline-based method when the amplitude threshold was lowered to $50 \mathrm{mV}$ - or even to 96 and
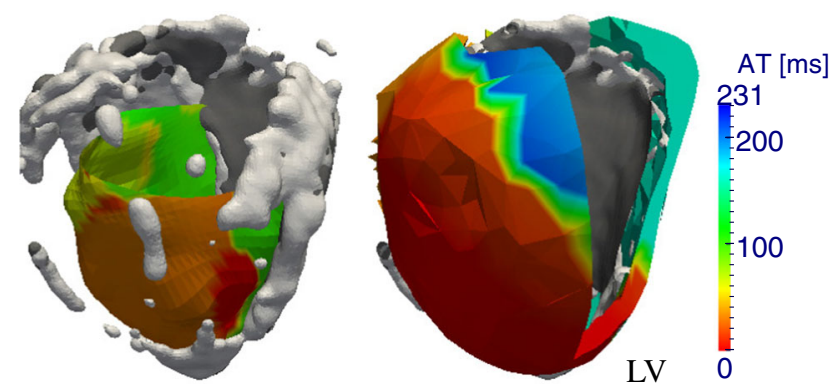

(a)
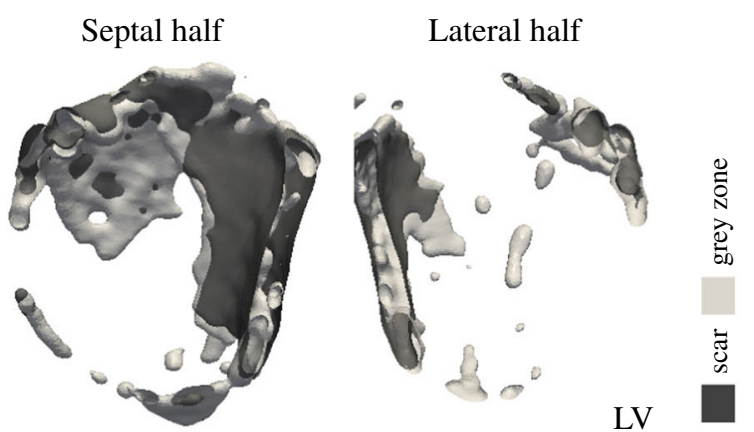

Greensite method
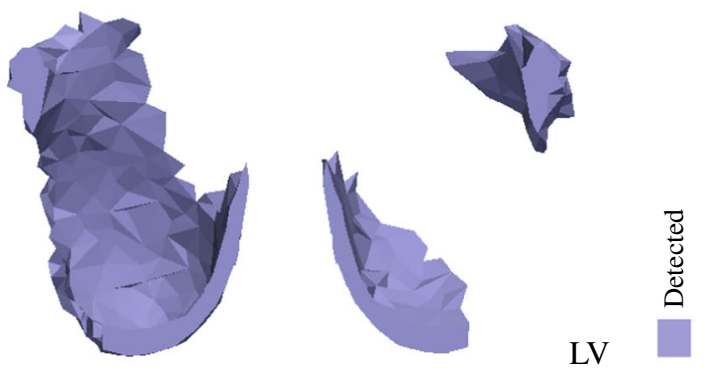

Spline-based
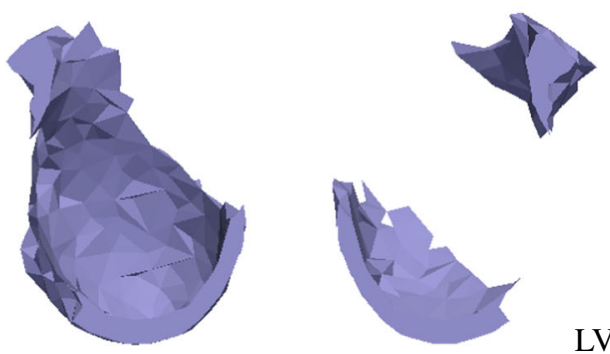

(b)

$92 \%$ when the threshold was lowered to $40 \mathrm{mV}$. Even though the increased specificities naturally came at the cost of strongly reduced sensitivities (25 and $24 \%$ for the $50 \mathrm{mV}$ threshold, 8 and $6 \%$ for the $40 \mathrm{mV}$ threshold), the fact that specificities rose to such levels indicates that very low-amplitude areas indeed belong to scar or grey zone tissue. 
Table 2 Statistical test on whether the difference between the maximum and the minimum TMV in a reconstructed TMV time curve can serve as a predictor for scar or grey zone ( $P$ positive condition) in the tissue

\begin{tabular}{ll}
\hline Greensite method & \\
$55.5 \%$ & Sensitivity (TP/P) \\
$70.4 \%$ & Specificity (TN/N) \\
Spline-based method & \\
$48.7 \%$ & Sensitivity (TP/P) \\
$65.8 \%$ & Specificity (TN/N) \\
\hline
\end{tabular}

Rates were calculated for nodes in the FEM mesh of the LV. TP: tissue is correctly predicted as scar or grey zone, TN: tissue is correctly predicted as healthy substrate

The authors intend to publish the dataset of this work in the EDGAR database [2] of the Consortium for Electrocardiograhpic Imaging at www.ecg-imaging.org.

\section{Discussion}

In this work, transmural distributions of TMVs were noninvasively imaged in a patient. The TMVs were evaluated in terms of their activation times against endocardially reconstructed isochrones as obtained from invasive MEA measurements. Further, TMVs were evaluated in terms of their action potential amplitudes against scar and grey zone from cardiac MR.

As in a previous work on ECG imaging of VT by Wang et al. [63] the results show good qualitative agreement between the non-invasively reconstructed and the invasively reconstructed activation time patterns (see Fig. 2c, d). A wavefront is rotating counterclockwise from the apico-basal perspective and performs a re-entry through the inferolaterally situated scar and grey zone tissue. Results with the Greensite method and the spline-based approach were very similar, which may be attributed to the underlying Tikhonov regularization in both methods. The exit point is close to the anterolateral extents of the grey zone (see also Fig. 1b in [7]) where it is suggested that a channel of healthy myocardium is situated in this area. However, solutions are smoothed out with respect to the isochrones in the EnSite ${ }^{\circledR}$ system, and the area of early activation is clearly drawn towards the base with an error of around $1 \mathrm{~cm}$ (see Table 1). This is despite the successful co-registration, which is reflected in a good correspondence between the invasively reconstructed signals on the shell and the scar tissue in the CMR-based model in [7]. The localization error is slightly greater than the error of 5-7 $\mathrm{mm}$ in the previous animal studies on VT [21, 22]. However, there are no localization errors available from previous VT studies in humans [54, 63].
While the activation patterns are very similar in their overall directions, the isochrones of the EnSite ${ }^{\circledR}$ system are in fact rather discrete, compared to the gradual activation times from ECG imaging. Further, they range only from 0 to $123 \mathrm{~ms}$, while results with the method by Greensite and the spline-based method range up to 231 and $227 \mathrm{~ms}$, respectively. This prolongation of activation in the reconstructed TMVs may be partially due to the choice of a smooth temporal and spatial basis in case of the Greensite method, which may lead to smoothed out wave fronts in the TMVs, and to the reduction of ECG data to knot points as well as spatial smoothing in the spline-based method. The prolongation and the rather discrete distribution of isochrones in the EnSite ${ }^{\circledR}$ reference are reflected in little linear correlation of only $66 \%$ for the method by Greensite in Table 1 or $63 \%$ for the spline-based method, and in a RMSE of 53 or $61 \mathrm{~ms}$. The evaluation of the robustness of results revealed a great sensitivity of the method by Greensite to its spatial regularization parameter $\lambda$ for the present case, while results were robust against changes in the truncation parameter $p$. Similarly, results with the spline-based method by Erem et al. were little sensitive to variations in the parameterization of its knots number. In effect, this shows that the automatic choice of its internal spatial regularization parameter with the L-curve method did not only lead to the best results in the study when results with manual corrections in the spatial regularization are discarded, but it also suggests that its automatic choice is quite robust. For the Greensite method, however, the L-curve failed to identify the optimal regularization factor by one order of magnitude. The resulting correction of this parameter after knowing the activation times from the noncontact mapping has deliberately and explicitly imposed a bias on the reported performance figures in Table 1 for the Greensite method. It has further likely led to a positive bias on the sensitivities and specificities for detecting scar or grey zone areas with the Greensite method as reported in Table 2. The analysis in Fig. 3 demonstrates the effect of this deliberate choice, and it also shows the effect of the (rather ineffectual) choice of $p=10$ and 14 knots. As a single case study, the robustness evaluation of the two methods and their parameterization in this work cannot be generalized. Still, for the Greensite method the failure of the L-curve method clearly demonstrates that alternative empirical or rule-based methods [48] need to be found to identify spatial regularization parameters reliably. The strong dependence of the solution on this parameter underlines the required precision and robustness of such methods.

According to some sources in the literature, the activation isochrones of the EnSite ${ }^{\circledR}$ system may well be considered to be an accurate enough reference for this evaluation study of ECG imaging: as stated in [36, 
Sect. 9.7.3.2] the EnSite ${ }^{\circledR}$ system has been shown to have good accuracy within $40 \mathrm{~mm}$ of the MEA catheter [28, 60], which is well within the range where results have been evaluated in the present study (the EnSite ${ }^{\circledR}$ shell had a diameter of $40 \mathrm{~mm}$, and the average registration error between the shell and the volumetric model of the LV was $3.3 \mathrm{~mm}$, with a standard deviation of $1.2 \mathrm{~mm}$ ).

However, the virtual electrograms which are used in the EnSite $^{\circledR}$ system to calculate activation times were shown to only have a mean correlation of around $80 \%$ with electrograms as measured with contact mapping [28], and a study by Abrams et al. [1] found significant reductions in the correlation, timing and amplitude of reconstructed electrograms from non-contact mapping compared to contact mapping when measuring at more than $40 \mathrm{~mm}$ from the MEA catheter. In this work, Abrams et al. even arrived at the conclusion that non-contact mapping cannot define areas of scar and low voltage accurately. These limitations of the invasive mapping may have contributed to the error in our results and may be attributed to the limited accuracy of MEA catheter location, the limited accuracy of the contoured-geometry approach and the use of an inverse approach to estimate the electrograms, which will lack the true morphology of contact electrograms [28].

Tikhonov-based solutions of the inverse problem of ECG may have unphysiological ranges and distributions, which is due to the effects of regularization, model resolution and model inaccuracies, which leads to offsets in the activation thresholds and again slight variations in the derived activation times. In this work, we scaled the reconstructed TMVs to a range of -85 to $15 \mathrm{mV}$ in each time step, which was necessary to obtain reasonable correlations of the activation times. The same was true for the amplitude threshold-based detection of scar tissue. Although it introduces assumptions on the physiological and pathophysiological range in space, the scaling to -85 to $15 \mathrm{mV}$ in each time step does not prevent scar tissue from having lower amplitudes in the TMV time courses. Still, the scaling may potentially lead to bias towards too large amplitudes in the solutions especially during the rather electrically silent part of the VT (between around 150 and $270 \mathrm{~ms}$ in the ECG): although it is valid for VT that at any time during a beat, depolarization is present as well as areas at resting membrane voltage, depolarization amplitudes may be lower when the wavefront propagates through scar tissue [35]. Excessive scaling at these time points may have caused activation peaks in the reconstructed time courses with a bias towards the end of the beat. In effect, this may have also contributed to the prolongation of activation with respect to the EnSite ${ }^{\circledR}$ ATs.

In this work, correlation was found between low amplitudes in the imaged TMVs and volumes of scar and grey zone in the CMR. The obtained sensitivity and specificity in
Table 2 are an indicator for the accuracy of the obtained inverse solutions, and they may motivate further research on methods for ECG imaging that make use of scar-related features, either as constraints in the regularization or as solution space for solvers of the inverse problem. While previous methods for the identification of scar [46, 64, 68, 71] have predominantly been designed for the reconstruction of sources of ST segment elevation, under the assumption that no sources from depolarization or repolarization exist during that time, the low-amplitude detection we present is based on depolarization effects during the entire ECG cycle. The results of the present study show the great potential of ECG imaging to overcome the problem of discriminating between scar, border zone and fibrotic tissue in CMR [29]. A discrimination between these electrophysiological substrate properties will lead to decisive advances in diagnosis [51], model-based therapy planning [8, 9, 32, 38, 59] and rehabilitation of the respective patients and thus lead to reduced burden of invasive procedures on the patient and lower treatment costs.

To conclude, the study underlines the ability of ECG imaging to produce activation times of ventricular electric activity-and to represent effects of scar tissue in the imaged transmembrane voltages.

Acknowledgments Rashed Karim was funded by the National Institute for Health Research (NIHR) Biomedical Research Centre based at Guy's and St Thomas' NHS Foundation Trust and King's College London. The views expressed are those of the author(s) and not necessarily those of the NHS, the NIHR or the Department of Health.

\section{Compliance with ethical standards}

Ethical approval All procedures performed in studies involving human participants were in accordance with the ethical standards of the institutional and/or national research committee and with the 1964 Helsinki Declaration and its later amendments or comparable ethical standards.

\section{References}

1. Abrams DJ, Earley MJ, Sporton SC, Kistler PM, Gatzoulis MA, Mullen MJ, Till JA, Cullen S, Walker F, Lowe MD, Deanfield JE, Schilling RJ (2007) Comparison of noncontact and electroanatomic mapping to identify scar and arrhythmia late after the Fontan procedure. Circulation 115(13):1738-1746

2. Aras K, Good W, Tate J, Burton B, Brooks D, Coll-Font J, Doessel O, Schulze W, Potyagaylo D, Wang L et al (2015) Experimental data and geometric analysis repository-EDGAR. J Electrocardiol 48(6):975-981

3. Berger T, Fischer G, Pfeifer B, Modre R, Hanser F, Trieb T, Roithinger FX, Stuehlinger M, Pachinger O, Tilg B, Hintringer F (2006) Single-beat noninvasive imaging of cardiac electrophysiology of ventricular pre-excitation. J Am Coll Cardiol 48:2045-2052

4. Burnes JE, Taccardi B, Rudy Y (2000) A noninvasive imaging modality for cardiac arrhythmias. Circulation 102:2152-2158 
5. Burnes JE, Taccardi B, Ershler PR, Rudy Y (2001) Noninvasive electrocardiogram imaging of substrate and intramural ventricular tachycardia in infarcted hearts. J Am Coll Cardiol 38:2071-2078

6. Burton BM, Tate JD, Erem B, Swenson DJ, Wang DF, Steffen M, Brooks DH, van Dam, PM, Macleod RS (2011) A toolkit for forward/inverse problems in electrocardiography within the scirun problem solving environment. In: Conference on Proceedings of IEEE Engineering in Medicine and Biology Society, pp 267-270

7. Chen Z, Relan J, Schulze W, Karim R, Sohal M, Shetty A, Ma YL, Ayache N, Sermesant M, Delingette H, Bostock J, Razavi R, Rhode K, Rinaldi A (2013) Simultaneous non-contact mapping fused with CMR derived grey zone to explore the relationship with ventricular tachycardia substrate in ischaemic cardiomyopathy. J Cardiovasc Magn Reson 15:64

8. Chen Z, Cabrera-Lozoya R, Rerlan J, Sohal M, Shetty A, Karim R, Delingette H, Gill J, Rhode K, Ayache N, Taggart P, Rinaldi CA, Sermesant M, Razavi R (2016) Biophysical modelling predicts ventricular tachycardia inducibility and circuit morphology: a combined clinical validation and computer modelling approach. J Cardiovasc Electrophysiol 27(7):851-860. doi:10.1111/jce. 12991

9. Chinchapatnam P, Rhode KS, Ginks M, Rinaldi CA, Lambiase P, Razavi R, Arridge S, Sermesant M (2008) Model-based imaging of cardiac apparent conductivity and local conduction velocity for diagnosis and planning of therapy. IEEE Trans Med Imaging 27:1631-1642

10. Coll-Font J, Burton BM, Tate JD, Erem B, Swenson DJ, Wang D, Brooks DH, Van Dam P, Macleod RS (2014) New additions to the toolkit for forward/inverse problems in electrocardiography within the SCIRun problem solving environment. Comput Cardiol 41:213-216

11. Coll-Font J, Potyagayo D, Schulze WH, Doessel O, Brooks DH (2015) Comparison of temporal dimensionality reduction methods for constrained inverse in cardiac electrical imaging. Comput Cardiol 42:237-240

12. Computational geometry algorithms library (www.cgal.org)

13. Ecabert O, Peters J, Schramm H, Lorenz C, von Berg J, Walker MJ, Vembar M, Olszewski ME, Subramanyan K, Lavi G, Weese J (2008) Automatic model-based segmentation of the heart in CT images. IEEE Trans Med Imaging 27:1189-1201

14. Erem B, Coll-Font J, Martinez Orellana R, Stovicek P, Brooks DH (2014) Using transmural regularization and dynamic modeling for non-invasive cardiac potential imaging of endocardial pacing with imprecise thoracic geometry. IEEE Trans Med Imaging 33:726-738

15. Friedman PA (2002) Novel mapping techniques for cardiac electrophysiology. Heart (British Cardiac Society) 87:575-582

16. Gabriel S, Lau RW, Gabriel C (1996) The dielectric properties of biological tissues: II. Measurements in the frequency range $10 \mathrm{~Hz}$ to $20 \mathrm{GHz}$. Phys Med Biol 41:2251-2269

17. Geselowitz DB, Miller TW (1983) A bidomain model for anisotropic cardiac muscle. Ann Biomed Eng 11:191-206

18. Ghanem RN, Jia P, Ramanathan C, Ryu K, Markowitz A, Rudy Y (2005) Noninvasive electrocardiographic imaging (ECGI): comparison to intraoperative mapping in patients. Heart Rhythm 2:339-354

19. Gornick CC, Adler SW, Pederson B, Hauck J, Budd J, Schweitzer J (1999) Validation of a new noncontact catheter system for electroanatomic mapping of left ventricular endocardium. Circulation 1999:829-835

20. Greensite F, Huiskamp G (1998) An improved method for estimating epicardial potentials from the body surface. IEEE Trans Biomed Eng 45:98-104

21. Han C, Pogwizd SM, Killingsworth CR, He B (2011) Noninvasive imaging of three-dimensional cardiac activation sequence during pacing and ventricular tachycardia. Heart Rhythm $8: 1266-1272$

22. Han C, Pogwizd SM, Killingsworth CR, He B (2012) Noninvasive reconstruction of the three-dimensional ventricular activation sequence during pacing and ventricular tachycardia in the canine heart. Am J Physiol Heart Circ 302:H244-H252

23. Hansen PC, O'Leary PC (1993) The use of the L-curve in the regularization of discrete ill-posed problems. SIAM J Sci Comput 14:1487-1503

24. He B, Li G, Zhang X (2003) Noninvasive imaging of cardiac transmembrane potentials within three-dimensional myocardium by means of a realistic geometry anisotropic heart model. IEEE Trans Biomed Eng 50:1190-1202

25. Hoekema R, Uijen G, van Oosterom A (1999) The number of independent signals in body surface maps. Methods Inf Med 38:119-124

26. Huiskamp G, Greensite F (1997) A new method for myocardial activation imaging. IEEE Trans Biomed Eng 44:433-446

27. Intini A, Goldstein RN, Jia P, Ramanathan C, Ryu K, Giannattasio B, Gilkeson R, Stambler BS, Brugada P, Stevenson WG, Rudy Y, Waldo AL (2005) Electrocardiographic imaging (ECGI), a novel diagnostic modality used for mapping of focal left ventricular tachycardia in a young athlete. Heart Rhythm 2:1250-1252

28. Kadish A, Hauck J, Pederson B, Beatty G, Gornick C (1999) Mapping of atrial activation with a noncontact, multielectrode catheter in dogs. Circulation 99(14):1906-1913

29. Karim R, Housden R, Balasubramaniam M, Chen Z, Perry D, Uddin A, Al-Beyatti Y, Palkhi E, Acheampong P, Obom S, Hennemuth A, Lu Y, Bai W, Shi W, Gao Y, Peitgen HO, Radau P, Razavi R, Tannenbaum A, Rueckert D, Cates J, Schaeffter T, Peters D, MacLeod R, Rhode K (2013) Evaluation of current algorithms for segmentation of scar tissue from late Gadolinium enhancement cardiovascular magnetic resonance of the left atrium: an open-access grand challenge. J Cardiovasc Magn Reson 15(1):105

30. Keller DUJ, Weber FM, Seemann G, Dössel O (2010) Ranking the influence of tissue conductivities on forward-calculated ECGs. IEEE Trans Biomed Eng 57:1568-1576

31. Krueger MW (2012) Personalized multi-scale modeling of the atria: heterogeneities, fiber architecture, hemodialysis and ablation therapy. KIT Scientific Publishing, Karlsruhe

32. Krueger MW, Seemann G, Rhode K, Keller DUJ, Schilling C, Arujuna A, Gill J, O'Neill MD, Razavi R, Dossel O (2013) Personalization of atrial anatomy and electrophysiology as a basis for clinical modeling of radio-frequency ablation of atrial fibrillation. IEEE Trans Med Imaging 32:73-84

33. Lai D, Sun J, Li Y, He B (2013) Usefulness of ventricular endocardial electric reconstruction from body surface potential maps to noninvasively localize ventricular ectopic activity in patients. Phys Med Biol 58:3897-3909

34. Liu C, Eggen MD, Swingen CM, Iaizzo PA, He B (2012) Noninvasive mapping of transmural potentials during activation in swine hearts from body surface electrocardiograms. IEEE Trans Med Imaging 31:1777-1785

35. Loewe A, Schulze WHW, Jiang Y, Wilhelms M, Luik A, Dössel O, Seemann G (2014) ECG-based detection of early myocardial ischemia in a computational model: impact of additional electrodes, optimal placement, and a new feature for ST deviation. BioMed Res Int 530352:1-11

36. Macfarlane PW, van Oosterom A, Pahlm O, Kligfield P, Janse M, Camm J (2010) Comprehensive electrocardiology. Springer, Berlin

37. MacLeod RS, Gardner M, Miller RM, Horacek BM (1995) Application of an electrocardiographic inverse solution to localize ischemia during coronary angioplasty. J Cardiovasc Electrophysiol 6:2-18 
38. McDowell KS, Zahid S, Vadakkumpadan F, Blauer J, MacLeod RS, Trayanova NA (2015) Virtual electrophysiological study of atrial fibrillation in fibrotic remodeling. PLoS ONE 10(2):e0117110

39. Messnarz B, Tilg B, Modre R, Fischer G, Hanser F (2004) A new spatiotemporal regularization approach for reconstruction of cardiac transmembrane potential patterns. IEEE Trans Biomed Eng 51(2):273-281

40. Müller HP, Godde P, Czerski K, Agrawal R, Feilcke G, Reither K, Wolf KJ, Oeff M (1999) Localization of a ventricular tachycardiafocus with multichannel magnetocardiography and three-dimensional current density reconstruction. J Med Eng Technol 23:108-115

41. Nash MP, Pullan AJ (2005) Challenges facing validation of noninvasive electrical imaging of the heart. Ann Noninvasive Electrocardiol 10:73-82

42. Nielsen BF, Lysaker M, Grottum P (2013) Computing ischemic regions in the heart with the bidomain model-first steps towards validation. IEEE Trans Med Imaging 32:1085-1096

43. Pfeifer B, Hanser F, Seger M, Fischer G, Modre-Osprian R, Tilg B (2008) Patient-specific volume conductor modeling for non-invasive imaging of cardiac electrophysiology. Open Med Inf J 2:32-41

44. Pop M, Sermesant M, Lepiller D, Truong M, McVeigh E, Crystal E, Dick A, Delingette H, Ayache N, Wright G (2009) Fusion of optical imaging and MRI for the evaluation and adjustment of macroscopic models of cardiac electrophysiology: a feasibility study. Med Image Anal 13:370-380

45. Potyagaylo D, Segel M, Schulze WHW, Dössel O (2013) Noninvasive localization of ectopic foci: a new optimization approach for simultaneous reconstruction of transmembrane voltages and epicardial potentials. FIMH Lect Notes Comput Sci 7945:166-173

46. Potyagaylo D, Cortes EG, Schulze WHW, Dössel O (2014) Binary optimization for source localization in the inverse problem of ECG. Med Biol Eng Comput 52:717-728

47. Potyagaylo D, Doessel O, Dam PV (2016) Influence of modeling errors on the initial estimate for nonlinear myocardial activation times imaging calculated with fastest route algorithm. IEEE Trans Biomed Eng PP(99), 1-1

48. Potyagaylo D, Schulze WHW, Dössel O (2012) A new method for choosing the regularization parameter in the transmembrane potential based inverse problem of ECG. Comput Cardiol 39:29-32

49. Rahimi A, Xu J, Wang L (2013) Lp-norm regularization in volumetric imaging of cardiac current sources. Comput Math Method Med 2013:10. doi:10.1155/2013/276478

50. Ramanathan C, Ghanem RN, Jia P, Ryu K, Rudy Y (2004) Noninvasive electrocardiographic imaging for cardiac electrophysiology and arrhythmia. Nat Med 10:422-428

51. Relan J, Chinchapatnam P, Sermesant M, Rhode K, Ginks M, Delingette H, Rinaldi CA, Razavi R, Ayache N (2011) Coupled personalization of cardiac electrophysiology models for prediction of ischaemic ventricular tachycardia. Interface Focus 1:396-407

52. Rhode KS, Hill DL, Edwards PJ, Hipwell J, Rueckert D, Sanchez-Ortiz G, Hegde S, Rahunathan V, Razavi R (2003) Registration and tracking to integrate $\mathrm{X}$-ray and MR images in an XMR facility. IEEE Trans Med Imaging 22(11):1369-1378

53. Rhode K, Ma Y, Housden J, Karim R, Rinaldi CA, Cooklin M, Gill J, O’Neill M, Schaeffter T, Relan J, Sermesant M, Delingette H, Ayache N, Krueger MW, Schulze W, Seemann G, Dössel O, Razavi R (2012) Clinical applications of image fusion for electrophysiology procedures. In: Proceedings of ISBI 2012. pp. 1435-1438. Barcelona

54. Sapp JL, Dawoud F, Clements JC, Horácek BM (2012) Inverse solution mapping of epicardial potentials quantitative comparison with epicardial contact mapping. Circ Arrhythm Electrophysiol 5:1001-1009

55. Schilling RJ, Peters NS, Davies DW (1998) Simultaneous endocardial mapping in the human left ventricle using a noncontact catheter. Circulation 98:887-898
56. Schulze WHW, Elies Henar F, Potyagaylo D, Loewe A, Stenroos M, Dössel O (2013) Kalman filter with augmented measurement model: an ECG imaging simulation study. FIMH Lect Notes Comput Sci 7945:200-207

57. Schulze WHW (2015) ECG imaging of ventricular activity in clinical applications. KIT Scientific Publishing, Karlsruhe

58. SCI Institute: (2015) http://www.scirun.org, SCIRun: a scientific computing problem solving environment. Scientific Computing and Imaging Institute (SCI)

59. Sermesant M, Chabiniok R, Chinchapatnam P, Mansi T, Billet F, Moireau P, Peyrat JM, Wong K, Relan J, Rhode K, Ginks M, Lambiase P, Delingette H, Sorine M, Rinaldi CA, Chapelle D, Razavi R, Ayache N (2012) Patient-specific electromechanical models of the heart for the prediction of pacing acute effects in CRT: a preliminary clinical validation. Med Image Anal 16:201-215

60. Thiagalingam A, Wallace EM, Boyd AC, Eipper VE, Campbell CR, Byth K, Ross DL, Kovoor P (2004) Noncontact mapping of the left ventricle: insights from validation with transmural contact mapping. PACE 27:570-578

61. Tilg B, Fischer G, Modre R, Hanser F, Messnarz B, Schocke M, Kremser C, Berger T, Hintringer F, Roithinger FX (2002) Modelbased imaging of cardiac electrical excitation in humans. IEEE Trans Med Imaging 21:1031-1039

62. Wang D, Kirby RM, Macleod RS, Johnson CR (2011) An optimization framework for inversely estimating myocardial transmembrane potentials and localizing ischemia. Proc Annu Int IEEE EMBS 2011:1680-1683

63. Wang Y, Cuculich PS, Zhang J, Desouza KA, Vijayakumar R, Chen J, Faddis MN, Lindsay BD, Smith TW, Rudy Y (2011) Noninvasive electroanatomic mapping of human ventricular arrhythmias with electrocardiographic imaging. Sci Transl Med 3:84

64. Wang L, Dawoud F, Yeung SK, Shi P, Wong KCL, Liu H, Lardo AC (2013) Transmural imaging of ventricular action potentials and post-infarction scars in swine hearts. IEEE Trans Med Imaging 32(4):731-747

65. Wang L, Wong K, Zhang H, Liu H, Shi P (2010) Statistical atlases and computational models of the heart. Lecture Notes in Computer Science, vol. 6364, chap. A statistical physiologicalmodel-constrained framework for computational imaging of subject-specific volumetric cardiac electrophysiology using optical imaging and MRI data, pp. 261-269

66. Wellens H, Brugada P, Stevenson W (1985) Programmed electrical stimulation of the heart in patients with life-threatening ventricular arrhythmias: What is the significance of induced arrhythmias and what is the correct stimulation protocol? Circulation 72:1-7

67. Wittkampf FH, Wever EF, Derksen R, Wilde AA, Ramanna H, Hauer RN, Robles de Medina EO (1999) Localisa: new technique for real-time 3-dimensional localization of regular intracardiac electrodes. Circulation 99:1312-1317

68. Xu J, Dehaghani AR, Gao F, Wang L (2014) Noninvasive transmural electrophysiological imaging based on minimization of total-variation functional. IEEE Trans Med Imaging 33(9):1860-1874

69. YingLiang M, Mistry U, Thorpe A, Housden RJ, Chen Z, Schulze WHW, Rinaldi CA, Razavi R, Rhode K (2013) Automatic electrode and CT/MR image co-localisation for electrocardiographic imaging. FIMH Lect Notes Comput Sci 7945:268-275

70. Yuan S, Blomstrom P, Pehrson S, Olsson SB (1991) Localization of cardiac arrhythmias: conventional noninvasive methods. Int $\mathrm{J}$ Cardiac Imaging 7:193-205

71. Zhou Z, Han C, Yang T, He B (2015) Noninvasive imaging of 3-dimensional myocardial infarction from the inverse solution of equivalent current density in pathological hearts. IEEE Trans Biomed Eng 62(2):468-476 
Walther Schulze studied electrical and computer engineering at the Karlsruhe Institute of Technology (KIT). He received a Ph.D. from the Institute of Biomedical Engineering of KIT in 2015.

Zhong Chen worked as a Research Student in Cardiovascular Imaging at the Division of Imaging Sciences of King's College London.

Jatin Relan worked at as a researcher in the Asclepios Project team at INRIA in Sophia Antipolis, France, where he received a Ph.D. in 2012.

Danila Potyagaylo studied applied mathematics at Kuban State University, Department of Mathematical Modeling, Krasnodar, Russia. He was Erasmus Mundus student of the M.Sc. course "Mathematical Modelling in Engineering: Theory, Numerics, Application"' in L'Aquila, Italy. He received a Ph.D. from the Institute of Biomedical Engineering at the Karlsruhe Institute of Technology (KIT) in 2016.

Martin Krueger studied electrical and computer engineering at the Karlsruhe Institute of Technology (KIT). He received a Ph.D. from the Institute of Biomedical Engineering of KIT in 2013 and works now at ABB AG, Corporate Research, Ladenburg, Germany.

Rashed Karim received a Ph.D. in Computer Science from Imperial College London in October 2009. He is currently a Research Fellow at King's College London and a Honorary Lecturer at Imperial College London.

Manav Sohal worked as a Clinical Research Fellow at King's College London. $\mathrm{He}$ is now EP Fellow at AZ Sint-Jan BruggeOostende AV in Brugge, Belgium, and a Complex EP Fellow as well as Clinical Fellow in Advanced Interventional EP at Brugmann University Hospital, Brussels, Belgium.

Anoop Shetty worked as a Research Student in Cardiovascular Imaging at the Division of Imaging Sciences of King's College London. He was later Electrophysiology Fellow at the Royal
Melbourne Hospital in Melbourne, Australia, and is now Consultant Cardiologist and Interventional Electrophysiologist at the Sheffield Teaching Hospitals NHS Foundation Trust.

YingLiang Ma was a Research Fellow at King's College London and is now a Senior Lecturer at the University of the West of England in Bristol, UK.

Nicholas Ayache is a Research Director at INRIA in Sophia Antipolis, France, where he is the Team Leader of the Asclepios Project team.

Maxime Sermesant received a Ph.D. in Control, Signal and Image Processing for his work in the EPIDAURE Project at INRIA, Sophia Antipolis, France. He currently works as a Researcher in the Asclepios Project team at INRIA in Sophia Antipolis.

Herve Delingette is a Researcher in the Asclepios Project team at INRIA in Sophia Antipolis.

Julian Bostock is a cardiac physiologist at Guy's \& St. Thomas, NHS Foundation Trust.

Reza Razavi is Head of the Division of Imaging Sciences and Biomedical Engineering at King's College London and Director of Research at King's Health Partners.

Kawal Rhode works at the Division of Imaging Sciences of King's College London. He is Professor of Biomedical Engineering at King's College London.

Christopher Rinaldi is Consultant Cardiologist at Guy's \& St. Thomas, NHS Foundation Trust, and Professor of Cardiac Electrophysiology at King's College London.

Olaf Dössel is the Director of the Institute of Biomedical Engineering at the Karlsruhe Institute of Technology (KIT). 